\title{
Ensuring optimal adherence to BCR-ABL1 tyrosine kinase inhibitor therapy for chronic myeloid leukemia
}

\author{
Mary Alma Welch, MMSC, PA-C, and \\ Elizabeth S. Kaled, RN, MS, CNC, NP-C, FNP-BC \\ University of Texas MD Anderson Cancer Center, Houston, Texas
}

The advent of BCR-ABL1 tyrosine kinase inhibitors (TKIs) for the treatment of chronic myeloid leukemia (CML) has dramatically changed the management of patients with CML. With continuous long-term TKI therapy, CML can be managed like a chronic condition, and most patients can expect to have a normal life expectancy. Given the prospect of lifelong therapy, however, issues related to adherence become particularly important and warrant greater attention since attainment of favorable long-term survival depends in large part on consistent, appropriate treatment administration over years, if not decades. As the multidisciplinary care team approach to cancer care has gained traction at academic centers and community practices, midlevel providers, including nurse practitioners and physician assistants, have taken on greater patient-related responsibilities. Midlevel providers have the potential to foster and maintain meaningful provider-patient relationships that may span years, and are well positioned to recognize and manage problems that patients may have with adherence. Here we discuss the importance of achieving and maintaining responses to TKI therapy, describe the clinical consequences of poor adherence to TKI therapy in $\mathrm{CML}$, and outline factors behind poor adherence. We also share strategies that we use at our center to improve adherence to long-term TKI therapy for CML.

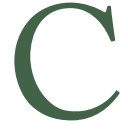

hronic myeloid leukemia (CML) is a blood cancer of uncontrolled myeloid cell growth. About 5,430 cases of CML were diagnosed in 2012..$^{1}$ Most patients are diagnosed in the early chronic phase (CP) of CML. Without treatment, $\mathrm{CML}$ progresses to the accelerated phase (AP) in 4-6 years and then to blast crisis (BC) and death in less than a year. ${ }^{2} \mathrm{CML}$, unlike most cancers, has a well-described molecular defect that is responsible for its pathogenesis: the unregulated activity of the BCR-ABL1 tyrosine kinase, which is produced as a result of an aberrant rearrangement of chromosomes 9 and 22 called the Philadelphia $(\mathrm{Ph})$ chromosome. $^{3}$ The discovery of the pathogenesis of CML led to the development of tyrosine kinase inhibitors (TKIs) that directly target the BCR-ABL1 protein and are highly effective against CML. Imatinib was the first TKI to be approved for treatment of CML, in 2001. In the phase 3 International Randomized

\footnotetext{
Manuscript received April 24, 2013; accepted April 29, 2013. Correspondence Mary Alma Welch, MMSC, PA-C, Department of Leukemia, Unit 428, University of Texas, MD Anderson Cancer Center, 1400 Holcombe Boulevard, Houston, TX 77030 (mawelch@mdanderson.org).

Disclosures The authors received financial support for medical editorial assistance from Novartis Pharmaceuticals Corporation. They have no other disclosures of conflicts of interest.
}

Study of Interferon and STI571 (IRIS), significant increases in rates of response and freedom from disease progression to $\mathrm{AP} / \mathrm{BC}$ were observed with imatinib compared with interferon- $\alpha$ plus cytarabine. ${ }^{4}$ Eight years of follow-up in the IRIS study have shown that responses to imatinib are durable and that imatinib significantly improves long-term survival. ${ }^{5-7}$

Since 2001, 2 additional TKIs, nilotinib and dasatinib, have been approved for first- and second-line treatment of CML, and 2 others, bosutinib and ponatinib, for second- or third-line use. Phase 3, randomized, controlled studies that compared nilotinib with imatinib (Evaluating $\mathrm{Ni}^{-}$ lotinib Efficacy and Safety in Clinical Trials Newly Diagnosed Patients [ENESTnd] $)^{8}$ and dasatinib with imatinib (Dasatinib versus Imatinib in Patients with Newly Diagnosed CML-CP [DASISION] $)^{9}$ showed that both nilotinib and dasatinib significantly improved rates of response compared with imatinib. In those studies, nilotinib and dasatinib lowered rates of disease progression to AP/BC compared with imatinib in the first-line setting. Continued follow-up of the ENESTnd and DASISION studies has shown that cytogenetic (c) 2013 Frontline Medical Communications 


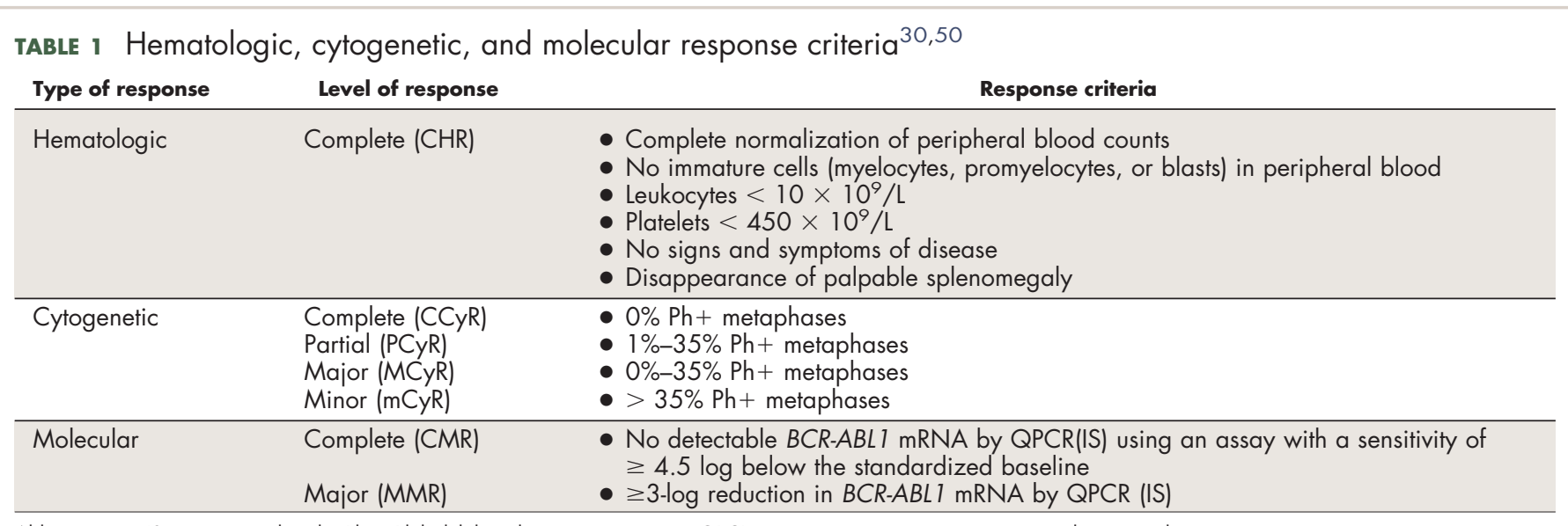

Abbreviations: IS, international scale; Ph+, Philadelphia chromosome-positive; QPCR, quantitative reverse transcription polymerase chain reaction.

and molecular responses, as well as protection against disease progression, are sustained at years $2,{ }^{10,11} 3,{ }^{12,13}$ and $4 .{ }^{14}$

BCR-ABL1 TKIs have transformed CML-CP from a progressive disease to a chronic disease - if managed effectively. The TKIs are oral agents that, at least by current standards, must be taken for life. Their efficacy depends on proper drug administration, which makes adherence to treatment a critical issue in patient management. Problems that may affect adherence, such as side effects, must be proactively addressed and corrected whenever possible to minimize the occurrence of unauthorized dose interruptions or treatment modifications that can diminish the clinical benefit of these agents.

In the past few decades, a greater emphasis has been placed on using a multidisciplinary team approach to manage cancer patients. ${ }^{15,16}$ As part of that evolution, midlevel providers, including nurse practitioners and physician assistants, are playing more central roles in patient care. As they develop comfortable relationships with patients, these health care practitioners are in prime position to monitor and recognize signs of waning adherence to TKI therapy. In this review, we discuss the importance of achieving and maintaining response to TKI therapy, describe the potential clinical consequences of poor adherence to TKI therapy in CML, and outline factors underlying poor adherence. We also share strategies, including those we have implemented in our center, that can be used to improve adherence to long-term TKI therapy for CML.

\section{Early response to TKI therapy predicts favorable long-term outcome}

Response to TKI therapy is based on the degree of improvement of hematologic, cytogenetic, and molecular parameters (Table 1). Numerous studies show that the achievement of early cytogenetic or molecular response to TKI therapy predicts a favorable long-term outcome. Most of this evidence is based on studies of imatinib, ${ }^{5-7,17-24}$ although there are corroborative results from studies involving nilotinib and dasatinib. ${ }^{18,25-27}$

Patients with rapid response generally have better long-term outcomes than do patients with slower or no response to TKI therapy. For example, one study showed that the probability of ever achieving complete cytogenetic response (CCyR) or major molecular response (MMR) decreased steadily with time if $\mathrm{CCyR}$ was not achieved by 3 , 6 , or 12 months. ${ }^{22}$ Two separate studies found that patients with optimal response (by European LeukemiaNet criteria) at 6 and 12 months had a survival advantage over patients with suboptimal response (or treatment failure) at those time points; at 18 months, however, patients with optimal response to treatment did no better than those with suboptimal response or treatment failure..$^{20,28}$

In particular, patients who achieve a reduction in $B C R-A B L 1$ transcript levels to $10 \%$ or less $(B C R-A B L 1$ $\leq 10 \%)$ at 3 months, which is roughly equivalent to a partial cytogenetic response (PCyR), were found to achieve significantly higher rates of deep molecular response and have significantly improved long-term progressionfree survival (PFS) and overall survival (OS) than did patients who had $B C R-A B L 1>10 \%$ at 3 months after the start of TKI therapy. For example, one study showed that patients on first-line imatinib with $B C R-A B L 1 \leq 10 \%$ at 3 months had significantly higher rates of OS and PFS at 8 years than did patients who had higher transcript levels (8-year OS: $93 \%$ vs $57 \%$, respectively, $P<.001$; 8-year PFS: $93 \%$ vs $57 \%, P<.001) .{ }^{29}$ These findings, along with similar findings from other studies, provide evidence that achievement of early molecular response has signifi- 
cant prognostic value in CML. The strength of the evidence led to a recent update to the National Comprehensive Cancer Network (NCCN) Clinical Practice Guidelines in Oncology (NCCN Guidelines), which now recommend the achievement of $B C R-A B L 1$ gene transcript level $\leq 10 \%$ as the 3 -month treatment response goal. ${ }^{30}$

Taken together, these findings underscore the importance of optimizing response to first-line treatment, particularly within the first year. Maintaining good adherence to a chronic medication during the first year of treatment can be challenging. Studies of adherence in patients with noncancer medical conditions (eg, diabetes, hypercholesterolemia, hypertension) requiring chronic medications show that about half of patients discontinue therapy within the first 6 months of treatment. ${ }^{31,32}$ The reason most often cited for not refilling a prescription was concern over side effects. ${ }^{31}$ Thus, to improve the likelihood of patient adherence, it is particularly important that the side effects of TKI therapy (including myelosuppression, fatigue, diarrhea, nausea, vomiting, fluid retention, rash, and headache) are well managed from the start of therapy.

\section{TKI therapy lowers the rate of disease progression to AP/BC}

The advanced stages of CML (AP and BC) are considerably more difficult to treat than is early-stage CML$\mathrm{CP}$. Treatment modalities that are effective in treating CML-CP, such as TKI therapy and stem cell transplantation, achieve lower rates of response and survival rates when used to treat CML-AP/BC. ${ }^{33,34}$ Furthermore, stem cell transplantation is associated with high morbidity, so its application is generally restricted to patients under 65 years of age. ${ }^{30}$ Because advanced CML poses therapeutic challenges, the stable maintenance of patients in CML$\mathrm{CP}$ has emerged as an overarching goal of modern CML management. ${ }^{35}$

The use of TKI therapy as first-line treatment in newly diagnosed patients significantly reduces the likelihood of disease progression. In the IRIS study, significantly more patients receiving imatinib were free from disease progression to $\mathrm{AP} / \mathrm{BC}$ at 18 months than patients receiving control treatment. With 3 years of follow-up in the ENESTnd study, significantly more of the patients receiving nilotinib were free from disease progression to $\mathrm{AP} / \mathrm{BC}$ at each year than were patients receiving imatinib. ${ }^{8,10,13}$ With 3 years of follow-up in the DASISION study, patients receiving dasatinib experienced fewer progressions to $\mathrm{AP} / \mathrm{BC}$ than did patients receiving imatinib, although the difference was not significant. ${ }^{9,11,12}$ Furthermore, patients with superior molecular or cytogenetic responses are significantly less likely to have disease progression than are patients with worse responses. For example, at the 5-year mark in the IRIS study, only $3 \%$ of patients who achieved CCyR at 12 months with imatinib had disease progression, compared with $19 \%$ of patients without a major cytogenetic response at 12 months. Similar progression outcome was found for patients who achieved both $\mathrm{CCyR}$ and MMR at 18 months compared with patients who did not. ${ }^{5}$ Taken together, these findings indicate that firstline TKI therapy in patients with newly diagnosed CML markedly delays disease progression, with the longest delay in patients who respond early.

\section{Poor adherence to TKI therapy in CML negatively affects treatment response and clinical status}

A significant correlation exists between level of adherence to TKI therapy in CML and clinical outcome. In the Adherence Assessment with Glivec: Indicators and Outcomes (ADAGIO) study, patients who achieved $\mathrm{CCyR}$ had a significantly higher adherence rate than patients who did not. ${ }^{36}$ Similarly, another study found that patients with $\leq 90 \%$ adherence were significantly less likely to achieve MMR or complete molecular response than were patients with $>90 \%$ adherence. ${ }^{37}$ Based on our experience, to reach $\geq 90 \%$ adherence, patients must take their medication for at least 27 days in a 30-day month. By this rule of thumb, missing 3-4 days of treatment in a 30-day month may lower the chances that a patient will achieve a good response (ie, CCyR or MMR).

Patients with poor adherence are also more likely to lose treatment response and eventually discontinue treatment. In one study, patients who had $\leq 85 \%$ adherence to imatinib were significantly more likely to lose $\mathrm{CCyR}$ at 2 years and to experience treatment failure than patients with $>85 \%$ adherence. ${ }^{38}$ More to the point, unexplained increases in $B C R-A B L 1$ transcript level by quantitative real-time polymerase chain reaction (QPCR) testing, loss of response to treatment as determined by blood counts or cytogenetic testing, or disease relapse marked by the manifestation of clinical symptoms may be signs of poor adherence. The effects of poor adherence underscore the importance of regular molecular monitoring of CML, as this allows health care providers to assess for early signs of waning adherence and/or inadequate response to treatment. The NCCN Guidelines recommend molecular monitoring of $B C R-A B L 1$ levels once every 3 months and evaluation for potential adherence problems when expected treatment response is not achieved at 3,12 , and 18 months. ${ }^{30}$ 
The overall consequences of poor adherence to TKI therapy extend beyond treatment response. Health economic studies show that patients with poorer adherence to TKI therapy incur significantly greater medical expenditures, including more inpatient admissions and greater medical costs. ${ }^{39,40}$ The implications of these observations are that poor adherence to TKI therapy results in overall clinical decline, most likely due to loss of response, subsequent treatment failure, and progression to advanced stages of disease.

\section{Factors underlying poor adherence}

Despite guidance from health care providers, patients may not fully appreciate the importance of adherence to TKI therapy in CML. In an interview-based study of patients with CML, patients reported both intentional and unintentional reasons for poor adherence to imatinib treatment. ${ }^{41}$ The primary reasons for a patient deciding not to take medication as prescribed were concern over side effects and interference of medication with social activities. Forgetfulness and prescribing error were the reasons that were cited for unintentional nonadherence, when a patient wanted but was not able to take medication. When the patients in this study were queried about nonadherence, they showed a lack of understanding of the potential clinical ramifications of nonadherence, with more than half affirming the belief that missing "the odd dose" would not affect their overall response to imatinib. These findings clearly indicate that patients could benefit from focused, specific guidance on how to successfully integrate chronic medications into their daily lives, so that taking them becomes part of their routine.

Some of the factors associated with poor adherence, such as younger age and shorter time on TKI therapy, are unmodifiable; however, the majority of factors can be modified, which offers midlevel providers the opportunity to intervene ${ }^{37,42,43}$ (Table 2). Modifiable factors associated with poor adherence include starting dose of the TKI, time between diagnosis and treatment initiation, number of concomitant medications, medication copayment, appearance of side effects during treatment, and symptom burden from side effects.

\section{Strategies to overcome problems with adherence}

Of the many ways to improve adherence, the most important is the establishment and maintenance of open lines of communication with patients. Also key is the discussion of adherence at every clinic visit. ${ }^{36,41,44} \mathrm{~A}$ survey study found that health care providers learned of nonadherence among CML patients more frequently through conversations with their patients than through other means, such as the frequency of prescription refills, ${ }^{45}$ underscoring the importance of connecting with patients on a regular basis.

The cost of long-term treatment can also have a significant bearing on adherence. Patients who are underinsured or have difficulty affording their medications may face considerable hurdles in maintaining optimal adherence to treatment over an extended period. They may not be aware that advocacy groups and pharmaceutical companies fund patient resources that provide financial assistance for health insurance payments and copayment obligations to those who cannot afford their medication (Table 3). Health care providers should inquire directly about issues related to finances, copayments, and insurance coverage that may affect the patient's access to medication, especially after any changes in insurance plans or coverage status. Addressing financial issues in a proactive manner can help prevent lapses in medication access that might affect patient health.

Another important principle is education, because many patients have little access to information about CML and are reluctant to ask questions. We are fortunate at our institution to have a dedicated patient education department that provides handouts on CML that present prescribing information in an abbreviated, patient-friendly format. Even without this type of resource, there are strategies that can be implemented by any practice. Review at every clinic visit the purpose of TKI therapy ${ }^{46}$ and how it works against the disease. Use plain language and terminology understandable to those with no medical background to explain the potential consequences of nonadherence. ${ }^{41}$ Communications from the health care provider should be tailored to the patient, taking into account the age of the patient, to ensure that information is relevant and understandable. Younger patients may be missing doses because of social pressures, such as a desire to conform or to avoid possible treatment-related side effects in social situations, and older patients may be missing doses because of forgetfulness or confusion. Understanding the fundamental reasons for poor adherence allows for targeted education to overcome specific barriers to treatment adherence. If possible, establish an adherence program in the practice that takes a proactive approach to applying adherence interventions, such as individual patient counseling, ${ }^{45}$ which has been shown to significantly improve patient adherence to TKI therapy in CML. Some specialty pharmacies may have their own programs that aim to improve treatment adherence and reduce medication waste. One specialty pharmacy chain has an oral chemotherapy cycle management program (CMP) that offers clinical support, dose monitoring, and early 
TABLE 2 Strategies to improve patient adherence to medication

Modifiable factors What midlevel providers can do

Starting dose of TKI therapy

- Work with the treating physician and the patient to select the most appropriate drug and dosage, taking into account patientdisease-, and treatment-related factors (eg, comorbidities, disease stage, safety profile).

Longer time between CML diagnosis and initiation of TKI therapy

- Work with the treating physician and the patient to develop a treatment plan at the time of or as soon as possible after diagnosis.
- Identify potential financial barriers to access to drugs and enroll patient in patient assistance programs if necessary.

- Emphasize to patient the importance of starting drug therapy in a timely manner.

Greater number of concomitant - Double-check that the correct dosage of drug is prescribed for medications

Wrong dosage of medication taken

CML.

- Verify at each clinic visit that the patient is taking the correct dosage (eg, "How many of these pills do you take each day?")

- Double-check that any new prescription, over-the-counter medications, or dietary supplements do not interact with TKI therapy.

High medication copayment

- Identify potential financial barriers to access to drugs and enroll patient in patient assistance programs if necessary.

\section{Frequency, severity, and/or persistence of adverse} events

- Develop a checklist of signs/symptoms of adverse events and review at each clinic visit.

- Emphasize to patient that even mild or common side effects should be reported.

- Ask what side effects the patient finds most bothersome (these might vary by patient).

- Manage side effects before they become severe or bothersome.

- Schedule frequent follow-up with patients who might be at higher risk of certain side effects (eg, patients with history of cardiovascular disorders, diabetes, or liver dysfunction).

Patient perceptions of therapy - Educate patient about CML disease background

- Ensure that patient understands what drug he/she is taking and why he/she is taking it.

- Set patient expectations for common side effects and likely treatment response.

- Track treatment response over time and celebrate with patient when specific milestones are achieved (eg, CCyR or MMR).

Patient forgetfulness

- Discuss adherence and drug administration (eg, "How many of these pills do you take each day?") at each visit.

- Make follow-up telephone calls between clinic visits to ensure that patient establishes good habits early on.

Prescribing error

- Write prescriptions in clear handwriting

- Use electronic prescriptions.

- Follow up with the pharmacy if an error is suspected.
- Learn the generic and brand names of the prescribed drug.

- Understand how and when to take the drug leg, with or without food, once or twice daily, number of pills per dose).

- Fill drug prescriptions as soon as possible.

- Begin drug treatment as directed by health care provider(s).
- Use a pill organizer to allocate medications on a daily or weekly basis.

- Inform the health care provider of any new prescription or over-the-counter medications, as well as dietary supplements.
- Inform the health care provider of changes in financial status or insurance coverage, especially if changes might affect access to treatment.

- Recognize the signs/symptoms of common side effects of treatment.

- Report all side effects, no matter how mild or infrequent.

- Ask about self-directed or nonprescription remedies to alleviate mild symptoms (eg, oatmeal baths for pruritus).
- Ask questions about CML, prescribed drugs, and prognosis.

- Understand patient responsibilities in CML treatmen (eg, take medications as directed, recognize and report side effects, keep all appointments, prepare for appointments - bring questions, pill bottles, diary of side effects).

- Set up reminders or prompts (eg, alarms, calendars).

- Use a pill organizer.

- Integrate medication into daily routine.

- Enlist the help of family members.

- Review medication dosage and administration if there is any doubt.

- Ask the pharmacist to contact the health care provider if necessary to review prescription.

- Know what drug you are taking by generic and brand name, and the dosage.

\begin{tabular}{lll}
\hline $\begin{array}{l}\text { Social events, } \\
\text { holidays/vacations }\end{array}$ & $\begin{array}{l}\text { - Explain clearly why continuous dosing is important for } \\
\text { treatment. }\end{array}$ & $\begin{array}{l}\text { - Plan ahead and ensure you have sufficient } \\
\text { medication for extended trips or trips overseas. }\end{array}$ \\
& treatment. & \\
trist the help of family or friends to play an active role in & Write a prescription or authorize a refill to ensure sufficient \\
medication for extended trips. & \\
\hline $\begin{array}{l}\text { Accidental (or intentional) } \\
\text { pregnancy }\end{array}$ & $\begin{array}{l}\text { - Educate patient about potential hazards that drugs may pose to } \\
\text { the fetus. }\end{array}$ & $\begin{array}{l}\text { - Use a reliable method of birth control to prevent } \\
\text { unintentional pregnancies. }\end{array}$ \\
& to immediately report any suspected pregnancies. & - Ask women of childbearing age about their use of adequate \\
and reliable birth control. & $\begin{array}{l}\text { Immediately report any suspected pregnancies to the } \\
\text { treating physician and report all medications to the } \\
\text { obstetrician. } \\
\text { - Discuss with the treating physician and the } \\
\text { obstetrician any desire to become pregnant before } \\
\text { becoming pregnant. }\end{array}$
\end{tabular}


TABLE 3 Selected patient financial assistance programs

\begin{tabular}{|c|c|}
\hline Program sponsor & Web address \\
\hline Ariad Pharmaceuticals & www.ariadpass.com \\
\hline Bristol-Myers Squibb & www.bms.com/products/Pages/programs.aspx \\
\hline Novartis Pharmaceuticals & www.novartisoncology.us/reimbursement/patt/index.jsp \\
\hline Pfizer & www.pfizerhelpfulanswers.com/pages/misc/Default.aspx \\
\hline
\end{tabular}

identification of side effects for patients with cancer. A study of this CMP found that patients who were enrolled in the program had significantly higher rates of persistence (ie, lower rates of discontinuation) and fewer hospitalizations associated with severe side effects than patients who were not enrolled. ${ }^{47}$

One simple way that we assess for adherence is to request patients bring their pill bottles to appointments, so that we can quickly determine whether the actual number of pills is consistent with the number of pills expected for that prescription period. By this method, we discovered that one of our patients had been taking too low a dose of nilotinib, despite verbal assurances that the correct dose was being taken; the number of pills she had brought to her appointment clearly showed that she had been taking nilotinib once daily instead of twice daily. Furthermore, we find that simply asking patients open-ended questions about their medication use (eg, "How many pills are you taking each day?") instead of leading questions (eg, "Are you taking two pills each day?") can be an effective way to determine whether patients are taking the proper dosage. We also recommend the use of a singlepage information card that contains information on dosage and administration (Figure 1), so that patients can have a simple visual guide to the proper use of their medication. This type of informational tool minimizes confusion, particularly for patients who are on multiple treatment regimens for concomitant conditions.

Because the occurrence of side effects is cited often as a reason for nonadherence, recognizing and managing side effects before they become impediments is vital to successful treatment. In our practice, we use a checklist of side effects to make sure that during clinic visits we describe to patients the more common and most clinically significant side effects. We also make it a point to learn what side effects patients consider most bothersome. This is a worthwhile effort, as a recent survey showed that health care providers in CML were off-target in naming what issues their patients consid- ered most important and underestimated the negative effect that symptom burden had on patient quality of life. ${ }^{48}$ Making assumptions about a patient's priorities when it comes to treatment and side effects is insufficient. A group at our institution has studied the utility of a telephone-based interactive voice response system in regularly assessing patient quality of life, including symptom burden, and found such a system to be successful and easy to implement, ${ }^{49}$ suggesting that side effects can be monitored at regular intervals without the need for frequent clinic visits.

There are also ways in which health care providers can empower patients to adhere to their medication regimens. In our practice, we help patients incorporate medication into their daily routine through the use of recommended prompts. ${ }^{41}$ For example, we help patients to set alarms or electronic calendars on personal devices (eg, cell phone or smartphone) to alert them when it is time to medicate. For patients who do not regularly carry electronic devices, we recommend they use a pill organizer. We make sure that patients have enough medication over weekends and holidays, since missing 3-4 days' worth of medication a month equivalent to a long weekend - may result in loss of response. When patients prepare to go on longer vacations, especially on trips overseas, we remind them to refill their prescriptions and double-check that they have enough medication for the entire time they will be away. We also enlist family members to take an active role in their loved one's treatment plan, so that patient care becomes a shared responsibility. We educate patients to recognize common side effects and to report all side effects, even the mild ones, when they occur. In this way, patients play an active role in the management of their side effects. We also make sure that patients learn about their medications, understand why they are taking them, and know both the generic and brand names. Having knowledge of their medical condition and treatment emboldens patients to ask questions about their overall treatment plan. Patients who 
A

\section{MY TREATMENT PLAN FOR CML}

This is the drug I am taking:
This is how much drug I must take:

\section{C}

\section{MY TREATMENT PLAN FOR CML}

This is the drug I am taking:
This is how much drug I must take:

\section{B}

\section{MY TREATMENT PLAN FOR CML}

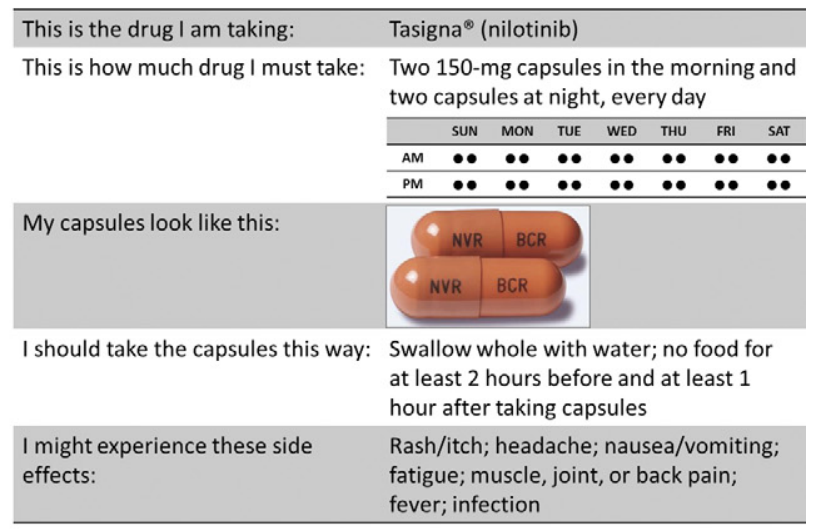

\section{D}

\section{MY TREATMENT PLAN FOR CML}

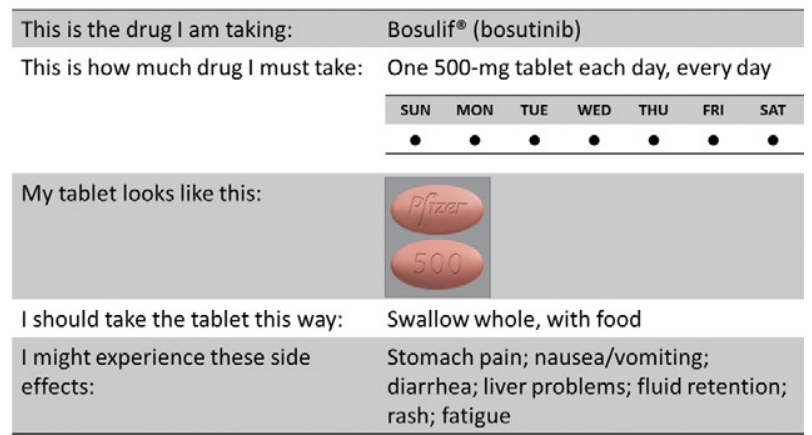

\section{E \\ MY TREATMENT PLAN FOR CML}

\begin{tabular}{l}
\hline This is the drug I am taking: \\
This is how much drug I must take:
\end{tabular}

FIGURE 1 Examples of single-page treatment plan cards for imatinib (A), nilotinib (B), dasatinib (C), bosutinib (D), and ponatinib (E).

feel a strong personal stake in their treatment and who fully understand the consequences of nonadherence are generally more motivated to do all they can to optimize long-term clinical outcome.

\section{Summary and conclusions}

The introduction of BCR-ABL1 TKI therapy has dramatically changed the way that patients with $\mathrm{CML}$ are managed, and the majority of patients can enjoy normal 
life expectancy. However, TKI therapy for CML may be lifelong, and ample evidence suggests that successful outcome depends in large part on optimal dosing and administration. It is critical that patients recognize the importance of consistent long-term adherence to their overall health.

As members of a multidisciplinary team, midlevel providers can play a prominent role in ensuring that adherence is optimized. By understanding the disease, the standard treatments, and the clinical evidence supporting their use in CML, midlevel providers can successfully educate their patients and advocate for the importance of adherence. Once common factors contributing to poor adherence are identified (eg, side effects, psychosocial issues), midlevel providers can proactively design strategies to address them before they affect treatment adherence. Finally, by empowering patients to make small changes that promote adherence (eg, establishing a medication routine, setting up reminders to take medication, planning ahead to account for holidays and vacations), midlevel providers can involve patients in maximizing the odds of successfully managing their CML and their overall long-term health.

\section{Acknowledgments}

We thank Anna Lau, PhD, and Patricia Segarini, PhD, of Percolation Communications LLC for their medical editorial assistance, for which Novartis Pharmaceuticals Corporation provided financial support.

\section{References}

1. American Cancer Society. Cancer Facts \& Figures. 2012. http:// www.cancer.org/acs/groups/content/@epidemiologysurveilance/ documents/document/acspc-031941.pdf. Accessed May 31, 2012.

2. Kalidas M, Kantarjian H, Talpaz M. Chronic myelogenous leukemia. JAMA. 2001;286(8):895-898.

3. Nowell PC, Hungerford DA. Chromosome studies on normal and leukemic human leukocytes. J Natl Cancer Inst. 1960;25:85-109.

4. O'Brien SG, Guilhot F, Larson RA, et al. Imatinib compared with interferon and low-dose cytarabine for newly diagnosed chronicphase chronic myeloid leukemia. N Engl J Med. 2003;348(11): 994-1004.

5. Druker BJ, Guilhot F, O'Brien SG, et al. Five-year follow-up of patients receiving imatinib for chronic myeloid leukemia. $N$ Engl J Med. 2006;355(23):2408-2417.

6. Hochhaus A, O'Brien SG, Guilhot F, et al. Six-year follow-up of patients receiving imatinib for the first-line treatment of chronic myeloid leukemia. Leukemia. 2009;23(6):1054-1061.

7. Deininger M, O'Brien SG, Guilhot F, et al. International randomized study of interferon vs STI571 (IRIS) 8-year follow up: sustained survival and low risk for progression or events in patients with newly diagnosed chronic myeloid leukemia in chronic phase (CML-CP) treated with imatinib [ASH abstract 1126]. Blood. 2009;114(22)

8. Saglio G, Kim DW, Issaragrisil S, et al. Nilotinib versus imatinib for newly diagnosed chronic myeloid leukemia. $N$ Engl J Med. 2010;362(24):2251-2259.

9. Kantarjian H, Shah NP, Hochhaus A, et al. Dasatinib versus imatinib in newly diagnosed chronic-phase chronic myeloid leukemia. N Engl J Med. 2010;362(24):2260-2270.

10. Kantarjian HM, Hochhaus A, Saglio G, et al. Nilotinib versus imatinib for the treatment of patients with newly diagnosed chronic phase, Philadelphia chromosome-positive, chronic myeloid leukaemia: 24-month minimum follow-up of the phase 3 randomised ENESTnd trial. Lancet Oncol. 2011;12(9):841-851.

11. Kantarjian HM, Shah NP, Cortes JE, et al. Dasatinib or imatinib in newly diagnosed chronic-phase chronic myeloid leukemia: 2-year follow-up from a randomized phase 3 trial (DASISION). Blood. 2012;119(5):1123-1129.

12. Hochhaus A, Shah NP, Cortes J, et al. Dasatinib versus imatinib (IM) in newly diagnosed chronic myeloid leukemia in chronic phase (CML-CP): DASISION 3-year follow-up [ASCO abstract 6504]. J Clin Oncol. 2012;30(suppl).

13. Larson RA, Hochhaus A, Hughes T, et al. Nilotinib vs imatinib in patients with newly diagnosed Philadelphia chromosomepositive chronic myeloid leukemia in chronic phase: ENESTnd 3-year follow-up. Leukemia. 2012;26(10):2197-2203.

14. Kantarjian HM, Kim D-W, Issaragrisil S, et al. Enestnd 4-year (y) update: continued superiority of nilotinib vs imatinib in patients (pts) with newly diagnosed Philadelphia chromosome-positive $(\mathrm{Ph}+)$ chronic myeloid leukemia in chronic phase (CML-CP) [ASH abstract 1676]. Blood. 2012;120(21).

15. Fennell ML, Das IP, Clauser S, Petrelli N, Salner A. The organization of multidisciplinary care teams: modeling internal and external influences on cancer care quality. J Natl Cancer Inst Monogr. 2010;2010(40):72-80.

16. Holloway S, Lord K, Bethelmie-Bryan B, et al. Managing chronic myeloid leukemia: a coordinated team care perspective. Clin Lymphoma Myeloma Leuk. 2012;12(2):88-93.

17. Alvarado Y, Kantarjian H, O'Brien S, et al. Significance of suboptimal response to imatinib, as defined by the European LeukemiaNet, in the long-term outcome of patients with early chronic myeloid leukemia in chronic phase. Cancer. 2009;115(16):37093718.

18. Jabbour E, Kantarjian H, O'Brien S, et al. The achievement of an early complete cytogenetic response is a major determinant for outcome in patients with early chronic phase chronic myeloid leukemia treated with tyrosine kinase inhibitors. Blood. 2011; 118(17):4541-4546;quiz 759.

19. Latagliata R, Breccia M, Carmosino I, et al. Complete cytogenetic response after 3 months is a very early indicator of good response to imatinib as front-line treatment in chronic myelogenous leukemia [ASH abstract 3783]. Blood. 2011;118(21).

20. Marin D, Milojkovic D, Olavarria E, et al. European LeukemiaNet criteria for failure or suboptimal response reliably identify patients with CML in early chronic phase treated with imatinib whose eventual outcome is poor. Blood. 2008;112(12):4437-4444.

21. Ohm L, Arvidsson I, Barbany G, Hast R, Stenke L. Early landmark analysis of imatinib treatment in CML chronic phase: less than $10 \%$ BCR-ABL by FISH at 3 months associated with improved long-term clinical outcome. Am J Hematol. 2012;87(8):760-765.

22. Quintas-Cardama A, Kantarjian H, Jones D, et al. Delayed achievement of cytogenetic and molecular response is associated with increased risk of progression among patients with chronic myeloid leukemia in early chronic phase receiving high-dose or standard-dose imatinib therapy. Blood. 2009;113(25):6315-6321.

23. Hehlmann R, Hanfstein B, Erben P, et al. The prognostic significance of early molecular and cytogenetic response for long-term progression-free and overall survival in imatinib-treated chronic myeloid leukemia (CML) [ASCO abstract 6510]. J Clin Oncol. 2012;30(suppl).

24. Hanfstein B, Muller MC, Hehlmann R, et al. Early molecular and cytogenetic response is predictive for long-term progression-free and overall survival in chronic myeloid leukemia (CML). Leukemia. 2012;26(9):2096-2102.

25. Hochhaus A, Saglio G, Chuah C, et al. Dasatinib and imatinibinduced reductions in BCR-ABL transcript levels below $10 \%$ at 3 months are associated with improved responses in patients with newly diagnosed chronic myeloid leukemia in chronic phase (CML-CP): analysis of molecular response kinetics in the DASISION trial [ASH abstract 2767]. Blood. 2011;118(21). 
26. Marin D, Hedgley C, Clark RE, et al. Predictive value of early molecular response in patients with chronic myeloid leukemia treated with first line dasatinib. Blood. 2012;120(2):291-294.

27. Nicolini FE, Hayette S, Labussiere H, et al. The month three major molecular response in chronic phase chronic myeloid leukemia on imatinib400, nilotinib and dasatinib is a major prognostic factor for failure-free and progression-free survival [ASH abstract 1684]. Blood. 2011;118(21):1684.

28. Kim D, Goh HG, Kim SH, et al. Comprehensive therapeutic outcomes of frontline imatinib mesylate in newly diagnosed chronic phase chronic myeloid leukemia patients in Korea: feasibility assessment of current ELN recommendation. Int $\mathrm{J} \mathrm{He}$ matol. 2012;96(1):47-57.

29. Marin D, Ibrahim AR, Lucas $\mathrm{C}$, et al. Assessment of $B C R-A B L 1$ transcript levels at 3 months is the only requirement for predicting outcome for patients with chronic myeloid leukemia treated with tyrosine kinase inhibitors. J Clin Oncol. 2012;30(3):232-238.

30. NCCN Clinical Practice Guidelines in Oncology. Chronic myelogenous leukemia. Version 3.2013. 2013. http://www.nccn.org/ professionals/physician_gls/pdf/cml.pdf. Accessed January 21, 2013.

31. Hugtenburg JG, Blom AT, Kisoensingh SU. Initial phase of chronic medication use; patients' reasons for discontinuation. $\mathrm{BrJ}$ Clin Pharmacol. 2006;61(3):352-354.

32. Yeaw J, Benner JS, Walt JG, Sian S, Smith DB. Comparing adherence and persistence across 6 chronic medication classes. $J$ Manag Care Pharm. 2009;15(9):728-740.

33. Martin MG, Dipersio JF, Uy GL. Management of the advanced phases of chronic myelogenous leukemia in the era of tyrosine kinase inhibitors. Leuk Lymphoma. 2009;50(1):14-23.

34. Saussele S, Lauseker M, Gratwohl A, et al. Allogeneic hematopoietic stem cell transplantation (allo SCT) for chronic myeloid leukemia in the imatinib era: evaluation of its impact within a subgroup of the randomized German CML Study IV. Blood. 2010;115(10):1880-1885.

35. Hehlmann R. How I treat CML blast crisis. Blood. 2012;120(4): 737-747.

36. Noens L, van Lierde MA, De Bock R, et al. Prevalence, determinants, and outcomes of nonadherence to imatinib therapy in patients with chronic myeloid leukemia: the ADAGIO study. Blood. 2009;113(22):5401-5411.

37. Marin D, Bazeos A, Mahon FX, et al. Adherence is the critical factor for achieving molecular responses in patients with chronic myeloid leukemia who achieve complete cytogenetic responses on imatinib. J Clin Oncol. 2010;28(14):2381-2388.

38. Ibrahim AR, Eliasson L, Apperley JF, et al. Poor adherence is the main reason for loss of $\mathrm{CCyR}$ and imatinib failure for chronic myeloid leukemia patients on long-term therapy. Blood. 2011; 117(14):3733-3736.

39. Wu EQ, Guerin A, Yu AP, et al. Retrospective real-world comparison of medical visits, costs, and adherence between nilotinib and dasatinib in chronic myeloid leukemia. Curr Med Res Opin. 2010;26(12):2861-2869

40. Wu EQ Johnson S, Beaulieu N, et al. Healthcare resource utilization and costs associated with non-adherence to imatinib treatment in chronic myeloid leukemia patients. Curr Med Res Opin. 2010;26(1):61-69.

41. Eliasson L, Clifford S, Barber N, Marin D. Exploring chronic myeloid leukemia patients' reasons for not adhering to the oral anticancer drug imatinib as prescribed. Leuk Res. 2011;35(5):626630.

42. St Charles M, Bollu VK, Hornyak E, et al. Predictors of treatment non-adherence in patients treated with imatinib mesylate for chronic myeloid leukemia [ASH abstract 2209]. Blood. 2009; 114(22).

43. Williams LA, Ault PS, Garcia-Gonzalez A, et al. Identifying high symptom burden in patients with chronic myeloid leukemia on tyrosine kinase inhibitor therapy [ASH abstract 3138]. Blood. 2011;118(21).

44. Schaffer SD, Yoon SJ. Evidence-based methods to enhance medication adherence. Nurse Pract. 2001;26(12):44, 50, 52, 54.

45. Guilhot F, Coombs J, Zernovak O, Szczudlo T, Rosti G. A global retrospective and physician-based analysis of adherence to tyrosine kinase inhibitor (TKI) therapies for chronic myeloid leukemia (CML) [ASH abstract 1514]. Blood. 2010;116(21).

46. Ruddy K, Mayer E, Partridge A. Patient adherence and persistence with oral anticancer treatment. CA Cancer J Clin. 2009;59(1):5666.

47. Khandelwal N, Duncan I, Ahmed T, Rubinstein E, Pegus C. Oral chemotherapy program improves adherence and reduces medication wastage and hospital admissions. J Natl Compr Canc Netw. 2012;10(5):618-625.

48. Efficace F, Breccia M, Saussele S, et al. Which health-related quality of life aspects are important to patients with chronic myeloid leukemia receiving targeted therapies and to health care professionals? GIMEMA and EORTC Quality of Life Group. Ann Hematol. 2012;90(9):1371-1381.

49. Garcia-Gonzalez A, Ault PS, Williams LA, et al. Interactive voice response system compliance in chronic myeloid leukemia population [ASCO abstract e19589]. J Clin Oncol. 2012;30(suppl).

50. Faderl S, Talpaz M, Estrov Z, Kantarjian HM. Chronic myelogenous leukemia: biology and therapy. Ann Intern Med. 1999; 131(3):207-219. 